\title{
Tensor Products of Quantized Tilting Modules
}

\author{
Henning Haahr Andersen \\ Matematisk Institut, Aarhus Universitet, DK-8000 Aarhus C, Denmark
}

Received October 25, 1991; in revised form February 16, 1992

\begin{abstract}
Let $U_{k}$ denote the quantized enveloping algebra corresponding to a finite dimensional simple complex Lie algebra $\mathfrak{E}$. Assume that the quantum parameter is a root of unity in $k$ of order at least the Coxeter number for $\mathfrak{E}$. Also assume that this order is odd and not divisible by 3 if type $G_{2}$ occurs. We demonstrate how one can define a reduced tensor product on the family $\mathscr{F}$ consisting of those finite dimensional simple $U_{k}$-modules which are deformations of simple $\mathfrak{L}$-modules and which have non-zero quantum dimension. This together with the work of Reshetikhin-Turaev and Turaev-Wenzl prove that $\left(U_{k}, \mathscr{F}\right)$ is a modular Hopf algebra and hence produces invariants of 3-manifolds. Also by recent work of Duurhus, Jakobsen and Nest it leads to a general topological quantum field theory. The method of proof explores quantized analogues of tilting modules for algebraic groups.
\end{abstract}

It was recently shown by Reshetikhin and Turaev [RT] that one can obtain invariants of 3-manifolds via quantum groups (see also [KM] and [TW]). In fact, [RT] contains a general procedure for the construction of invariants from modular Hopf algebras, and it was proved that the quantum group for $s l_{2}$ is a modular Hopf algebra. Moreover, in [TW] it is proved that the quantum groups corresponding to root systems of classical types are quasi-modular Hopf algebras and that such an algebra similarly produces invariants of 3-manifolds.

The purpose of this paper is to prove that a quantum group corresponding to any finite root system is a modular Hopf algebra (actually we only check two of the axioms since the remaining ones are verified in [TW]). More specifically, we prove this over any field of characteristic zero in which the quantum parameter is a root of unity of odd order $l$ bigger than the Coxeter number for the root system in question (if the root system involves type $G_{2}$, we assume $l$ also to be prime to 3 ).

In another recent (and related) development Durhuus, Jakobsen and Nest, [DJN], have demonstrated (generalising the results of Turaev and Viro [TV]) how one may obtain a topological quantum field theory from the rather general setup of an associative algebra with a distinguished finite set of irreducible representations 
equipped with a "nice" tensor product. Our theorem shows that the quantized enveloping algebras at a root of unity form a large class of examples where this setup is realized. (Most of the properties were already established in loc. cit. Our contribution is the existence of an associative tensor product which preserves semi-simplicity on the distinguished set of representations. (For quantum $s l_{2}$ this was shown in [RT].))

A key ingredient in our proof is the use of tilting modules for quantum groups. These modules are defined exactly as Donkin defines tilting modules for algebraic groups [D2] (see also [R]). In the last section which is not directly related to the above result, we prove some additional properties of quantized tilting modules. In particular, we find that the quantum analogue of two conjectures by Donkin are easy consequences of the results in [APW 1-2]. We view this as evidence in favour of the modular conjectures.

I would like to thank H.P. Jakobsen and V. Turaev for bringing the problem studied here to my attention and for stimulating my interest in it. Also I want to thank S. Donkin for selling me his tilting modules and for some useful remarks which simplified the proof of the main result.

Some time after this paper was submitted I received S. Gelfand and D. Kazhdan's preprint, "Examples of Tensor Categories," which contains results similar to our main theorem for the case where the quantum parameter has prime order.

\section{Quantum Groups at a Root of Unity}

Let $\mathfrak{L}$ denote a simple complex Lie algebra and let $U^{\prime}$ denote the corresponding quantized enveloping algebra over $\mathbb{Q}(v), v$ an indeterminate. Set $A=\mathbb{Z}\left[v, v^{-1}\right]$ and let $U_{A}$ denote the Lusztig $A$-form of $U^{\prime}$, see [L1].

Fix now a field $k$ and a primitive $l^{\text {th }}$ root of unity $\zeta \in k$. We shall assume that $k$ has characteristic zero, that $l$ is prime to the non-zero entries of the Cartan matrix for $\mathfrak{L}$ and that $l$ is bigger than the Coxeter number. The assumption on $k$ is mostly for convenience whereas there are several reasons - some of them built-in - for the restrictions on $l$.

The quantum group we shall consider throughout this paper is $U_{k}=U_{A} \otimes_{A} k$. Here $k$ is made into an $A$-algebra by specializing $v$ to $\zeta$.

We now recall from [APW1] some of the key results which we will need about finite dimensional representations of $U_{k}$. Most of these results are analogues of theorems in the modular representation theory of algebraic groups, see Part II of [J].

We shall use the notation from [APW1]. Recall in particular that $\mathscr{C}_{k}$ is the category of integrable $U_{k}$-modules and that it contains all finite dimensional modules of type 1 . We have a triangular decomposition $U_{k}=U_{k}^{-} U_{k}^{0} U_{k}^{+}$, and the induction functor from $\mathscr{C}_{k}^{-}$(the category of integrable $U_{k}^{-} U_{k}^{0}$-modules) to $\mathscr{C}_{k}$ is denoted $H_{k}^{0}$. From [AW, Theorem 2.5] we recall that the derived functors $H_{k}^{i}$ vanish for $i>N$, where $N$ is the number of positive roots. For a dominant weight $\lambda \in X^{+}$we shall in this paper use the notation

$$
V_{k}(\lambda)=H_{k}^{N}\left(w_{0} \cdot \lambda\right)
$$

where $w_{0}$ is the largest element in the Weyl group $W$. By Serre duality [AW, Theorem 3.2] we see that

$$
V_{k}(\lambda) \cong H_{k}^{0}\left(-w_{0} \lambda\right)^{*}
$$


Then the irreducible module $L_{k}(\lambda)$ is the socle of $H_{k}^{0}(\lambda)$ as well as the head of $V_{k}(\lambda)$.

From Kempf's vanishing theorem [AW, Theorem 5.3] it follows that $V_{k}(\lambda)$ and $H_{k}^{0}(\lambda)$ are deformations of the corresponding constructions for the algebraic group with Lie algebra $\mathfrak{L}$. To be specific, we have $V_{k}(\lambda) \cong H_{A}^{N}\left(w_{0} \cdot \lambda\right) \otimes_{A} k$ and $H_{k}^{0}(\lambda) \cong H_{A}^{0}(\lambda) \otimes_{A} k$, where $H_{A}^{N}\left(w_{0} \cdot \lambda\right) \otimes_{A} \mathbb{C} \cong H_{A}^{0}(\lambda) \otimes_{A} \mathbb{C}$ is the irreducible $\mathbb{Q}$ module with highest weight $\lambda$. Here $\mathbb{C}$ is the $A$-algebra obtained by specializing $v$ to 1 .

We shall need the following (special case of the) linkage principle for quantum groups at a primitive $l^{\text {th }}$ root of 1 , see [APW1, Theorem 8.1] and [T].

If $\lambda, \mu \in X^{+}$and $L_{k}(\mu)$ is a composition factor of $H_{k}^{0}(\lambda)$,

then $\mu$ is strongly linked to $\lambda$.

The standard argument [CPS vdK ], [J, II.4.10] shows that Kempf's vanishing theorem [AW, Theorem 5.3] has the following consequence:

Let $\lambda \in X^{+}$and let $M \in \mathscr{C}_{k}$. If $\operatorname{Ext}_{\mathscr{C}_{k}}^{i}\left(M, H_{k}^{0}(\lambda)\right) \neq 0$ for some $i \geqq 0$, then $M$ has a weight $\mu$ with $\mu \geqq \lambda$. If $i>0$, then $\mu>\lambda$.

A particular case of (1.3) is

Let $\lambda, \mu \in X^{+}$. Then $\operatorname{Ext}_{\mathscr{C}_{k}}^{i}\left(V_{k}(\lambda), H_{k}^{0}(\mu)\right)=0$ for $i>0$.

Another standard argument [J, II.6.17] gives that (1.2) and (1.4) imply

Let $\lambda, \mu \in X^{+}$. If $L_{k}(\lambda)$ and $L_{k}(\mu)$ both are composition

factors of an indecomposable module $M \in \mathscr{C}_{k}$, then $\lambda \in W_{l} \cdot \mu$.

This linkage principle allows us to decompose any module from $\mathscr{C}_{k}$ into summands corresponding to $W_{l}$-orbits:

Denote by $C$ the first dominant alcove, i.e.

$$
C=\left\{\lambda \in X^{+} \mid\left\langle\lambda+\rho, \alpha^{v}\right\rangle<l \text { for all } \alpha \in R_{+}\right\}
$$

and set

$$
\bar{C}=\left\{\lambda \in X \mid 0 \leqq\left\langle\lambda+\rho, \alpha^{\vee}\right\rangle \leqq l \text { for all } \alpha \in R_{+}\right\} .
$$

Then $\bar{C}$ is a fundamental domain for $W_{l}$. For $\mu \in \bar{C}$ and $M \in \mathscr{C}_{k}$, we set

$$
\begin{aligned}
p_{\mu}(M)= & \text { the maximal submodule of } M \text { whose composition } \\
& \text { factors have highest weights in } W_{l} \cdot \mu .
\end{aligned}
$$

With this notation at hand we can reformulate (1.5) as follows, cf. [J, II.7.3]:

$$
\text { For } M \in \mathscr{C}_{k} \text { we have } M=\bigoplus_{\mu \in \bar{C}} p_{\mu}(M) \text {. }
$$

\section{Tilting Modules}

In this section we define tilting modules for quantum groups and deduce their first properties. 
Definition 2.1. A finite dimensional module $M \in \mathscr{C}_{k}$ is said to have a good filtration (respectively a Weyl filtration) if there exists a sequence

$$
0=F_{0} \subset F_{1} \subset \ldots \subset F_{v}=M
$$

of submodules with $F_{i} / F_{i-1} \cong H_{k}^{0}\left(\lambda_{i}\right)$ (respectively $V_{k}\left(\lambda_{i}\right)$ ) for some $\lambda_{i} \in X^{+}$, $i=1, \ldots, r$.

Note that because of (1.1), a module $M$ has a good filtration if and only if $M^{*}$ has a Weyl filtration (and vice versa). Let us also record here the following consequence of (1.3):

If $M$ has a good filtration as in 2.1, then this filtration

can be rearranged so that $i<j$ whenever $\lambda_{i}<\lambda_{j}$.

Of course there is an analogous statement about Weyl filtrations.

It is easy to see that a direct summand of a module with a good filtration has a good filtration. The following result is much deeper.

Theorem 2.3. If $M_{1}$ and $M_{2}$ are modules with good filtration, then so is $M_{1} \otimes_{k} M_{2}$.

Proof. In the case where $l$ is a prime power, this result is recorded in [APW1, Corollary 5.14]. The proof there relies on the corresponding theorem for reductive algebraic groups in characteristic $p>0$, see [W, D1, M].

In the general case the result follows from Lusztig's work on canonical bases, see [L2, Sect. 4] (this was pointed out to me by S. Donkin. For details see [P]). Of course, we could also just quantize [W] (since we are assuming $l>h$ ).

Definition 2.4. A (partial) tilting module for $U_{k}$ is a module $M \in \mathscr{C}_{k}$ which has both a good filtration and a Weyl filtration.

This definition is the exact analogue of Donkin's definition of tilting modules for algebraic groups, see [D2]. Like in Donkin's work we have the following version of Ringel's theorem on the existence and uniqueness of indecomposable tilting modules [R].

Theorem 2.5. i) For each $\lambda \in X^{+}$there exists an indecomposable tilting module $Q(\lambda)$ which has unique highest weight $\lambda$. Furthermore, $\lambda$ occurs with multiplicity 1 as a weight of $Q(\lambda)$.

ii) The modules $Q(\lambda), \lambda \in X^{+}$, form a complete set of inequivalent indecomposable tilting modules.

Corollary 2.6. Let $M$ be a tilting module. Then there exist uniquely determined $a_{\lambda}(M) \in \mathbb{N}, \lambda \in X^{+}$, such that $M=\bigoplus_{\lambda \in X^{+}} Q(\lambda)^{\oplus a_{\lambda}(M)}$. In fact, $a_{\lambda}(M)$ is determined by the character $\operatorname{ch} M$ of $M$.

Remark 2.7. Since $U_{k}$ is not co-commutative, we do not always have $M_{1} \otimes M_{2} \cong M_{2} \otimes M_{1}$. However, if $M_{1}$ and $M_{2}$ both are tilting modules, then $M_{1} \otimes M_{2} \cong M_{2} \otimes M_{1}$ (because by Corollary 2.6 we get $a_{\lambda}\left(M_{1} \otimes M_{2}\right)=$ $a_{\lambda}\left(M_{2} \otimes M_{1}\right)$ for all $\left.\lambda\right)$.

\section{Endomorphisms of Tilting Modules}

In this section we shall prove that the quantum traces of all endomorphisms of certain tilting modules vanish. As we shall see in the next section, this is exactly 
what is needed in order to apply the constructions in [RT] and [DJN] to quantum groups.

If $f$ is an endomorphism of a finite dimensional vector space, we denote by $\operatorname{tr}(f)$, the trace of $f$.

Definition 3.1. Let $M \in \mathscr{C}_{k}$ be a finite dimensional module and let $\varphi$ be an endomorphism of $M$. By the quantum trace, $\operatorname{tr}_{q}(\varphi)$, we understand $\operatorname{tr}\left(K_{2 \rho} \varphi\right)$, where $K_{2 \rho}=\prod_{\beta} K_{\beta}$, the product running over all positive roots.

By the quantum dimension, $\operatorname{dim}_{q} M$, we understand the quantum trace of the identity on $M$, i.e. $\operatorname{dim}_{q} M=\operatorname{tr}\left(K_{2 \rho}\right)$.

It is clear from the definition how to obtain $\operatorname{dim}_{q} M$ once the character of $M$ is known. Let us illustrate this in the case $M=V_{k}(\lambda)$ :

For $\lambda \in X$ we denote by $\chi(\lambda) \in \mathbb{Z}[X]$ the usual Weyl character, i.e. $\chi(\lambda)=\sum_{w \in W}(-1)^{l(w)} e^{w(\lambda+\rho)} / \sum_{w \in W}(-1)^{l(w)} e^{w(\rho)}$. When $\lambda \in X^{+}$, we have $\chi(\lambda)=\operatorname{ch} V_{k}(\lambda)$.

From this we obtain

$$
\operatorname{dim}_{q} V_{k}(\lambda)=\prod_{\beta>0} \frac{\zeta^{d_{\beta}\left\langle\lambda+\rho, \beta^{\vee}\right\rangle}-\zeta^{-d_{\beta}\left\langle\lambda+\rho, \beta^{\vee}\right\rangle}}{\zeta^{d_{\beta}\left\langle\rho, \beta^{\vee}\right\rangle}-\zeta^{d_{\beta}\left\langle\rho, \beta^{\vee}\right\rangle}} .
$$

(Here $d_{i} \in\{1,2,3\}$ are the integers used to symmetrize the Cartan matrix, and if a positive root $\beta$ is the $W$-conjugate of the simple $\alpha_{i}$, then $d_{\beta}=d_{i}$.)

Recall that $\lambda \in X$ is called $l$-singular if $\left\langle\lambda+\rho, \beta^{\vee}\right\rangle$ is divisible by $l$ for some root $\beta$. Otherwise $\lambda$ is called $l$-regular. From (3.2) we get in particular

$$
\text { If } \lambda \in X^{+}, \text {then } \operatorname{dim}_{q} V_{k}(\lambda) \neq 0 \text { iff } \lambda \text { is } l \text {-regular . }
$$

With the notation from Corollary 2.6 we can now state

Theorem 3.4. Let $M$ be a tilting module with $a_{\lambda}(M)=0$ for all $\lambda \in C$. Then for any endomorphism $f$ of $M$ we have $\operatorname{tr}_{q}(f)=0$.

To prove this theorem, let us first make a couple of reductions. First of all, it is clear from Corollary 2.6 that it is enough to verify the theorem in the case $M=Q(\lambda), \lambda \in X^{+} \backslash C$. Secondly, we observe that if $f$ is nilpotent, so is $K_{2 \rho} f$ (because our modules are direct sums of their weight spaces) and hence $\operatorname{tr}_{q}(f)=0$ for all nilpotent $f$. Now any endomorphism of the indecomposable module $Q(\lambda)$ is equal to a constant plus a nilpotent endomorphism. Hence the theorem reduces to

Proposition 3.5. Let $\lambda \in X^{+}$. Then $\operatorname{dim}_{q} Q(\lambda)=0$ iff $\lambda \notin C$.

We shall first observe that Proposition 3.5 is clear for $\lambda l$-singular: By (1.5) it follows immediately that if $V_{k}(\mu)$ occurs in a Weyl filtration of $Q(\lambda)$ and $\lambda$ is $l$-singular, then $\mu$ is also $l$-singular. But then $\operatorname{dim}_{q} V_{k}(\mu)=0$ by (3.3) and the proposition follows in this case.

The idea is now to obtain the $l$-regular case by translation. As a preparation we need the following elementary lemma:

Lemma 3.6. Let $E, M \in \mathscr{C}_{k}$ be finite dimensional and suppose $\operatorname{tr}_{q}(f)=0$ for all $f \in \operatorname{End}_{U_{k}}(M)$. Then $\operatorname{tr}_{q}(\varphi)=0$ for all $\varphi \in \operatorname{End}_{U_{k}}(E \otimes M)$. In particular, $\operatorname{dim}_{q} Q=0$ for all summands $Q$ of $E \otimes M$. 
Proof. Let $e_{1}, \ldots, e_{r}$ be a basis for $E$ and denote by $e_{1}^{*}, \ldots, e_{r}^{*}$, respectively $e_{1}^{* *}, \ldots, e_{r}^{* *}$ the dual (respectively double dual) basis in $E^{*}$ (respectively $E^{* *}$ ). The map $c: E \rightarrow E^{* *}$ which takes $e$ into $\left(h \rightarrow h\left(K_{-2 \rho} e\right), h \in E^{*}\right)$ is a $U_{k}$-homomorphism (because the square of the antipode on $U_{k}$ is conjugation by $K_{-2 \rho}$ ). Also the two maps

$$
\begin{array}{ll}
k \stackrel{i}{\rightarrow} E^{*} \otimes E^{* *} \quad \text { and } & E^{*} \otimes E \stackrel{\pi}{\rightarrow} k \\
1 \rightarrow \sum_{i} e_{i}^{*} \otimes e_{i}^{* *} & e_{i}^{*} \otimes e_{j} \rightarrow \delta_{i j}
\end{array}
$$

are $U_{k}$-homomorphisms.

So if $\varphi \in \operatorname{End}_{U_{k}}(E \otimes M)$, then the composite

$$
\tilde{\varphi}: M \stackrel{i \otimes 1}{\longrightarrow} E^{*} \otimes E^{* *} \otimes M \stackrel{1 \otimes c^{-1} \otimes 1}{\longrightarrow} E^{*} \otimes E \otimes M \stackrel{1 \otimes \varphi}{\longrightarrow} E^{*} \otimes E \otimes M \stackrel{\pi \otimes 1}{\longrightarrow} M
$$

is in $\operatorname{End}_{U_{k}}(M)$. It is an elementary exercise to check that

$$
\operatorname{tr}_{q}(\tilde{\varphi})=\operatorname{tr}_{q}(\varphi)
$$

The lemma follows.

Recall now that once we have the linkage principle $(1.5-6)$, we can define translation functors, $T_{\lambda}^{\mu}$ for $\lambda, \mu \in \bar{C}$, as follows: For $M \in \mathscr{C}_{k}$ we set $T_{\lambda}^{\mu} M=p_{\mu}(M \otimes E)$, where $E$ is a finite dimensional $U_{k}$-module with highest weight $w(\mu-\lambda)$. Here $w \in W$ is chosen so that $w(\mu-\lambda) \in X^{+}$. Usually one takes $E=L_{k}(w(\mu-\lambda))$, but actually any other module with $w(\mu-\lambda)$ as its unique highest weight will do just as well. We shall use the tilting module, i.e. we define

$$
T_{\lambda}^{\mu} M=p_{\mu}(M \otimes Q(w(\mu-\lambda))) .
$$

The advantage of replacing the simple module by the tilting module is that it is now immediately clear (by Theorem 2.3) that

$T_{\lambda}^{\mu}$ takes tilting modules to tilting modules .

We are now ready for the

Proof of Proposition 3.5. By the above observations we may assume that the highest weight of the tilting module in question is $l$-regular, i.e. equal to $w \cdot \lambda$ for some $\lambda \in C$ and $w \in W_{l} \backslash 1$. Then we choose $\mu \in \bar{C}$ such that

$$
\operatorname{stab}_{W_{l}}(\mu)=\{1, s\} \text { and } w s \cdot \lambda<w \cdot \lambda .
$$

(This means that $w \cdot \mu$ lies on a unique wall in the lower closure of $w \cdot C$.)

Since we already know that $\operatorname{dim}_{q} Q(w \cdot \mu)=0$, we see from Lemma 3.6 that also all the summands of $T_{\mu}^{\lambda}(Q(w \cdot \lambda)$ have $q$-dimension equal to zero. However, it is obvious that $w \cdot \lambda$ is the (unique) highest weight of $T_{\mu}^{\lambda} Q(w \cdot \mu)$, and hence $Q(w \cdot \lambda)$ is a summand. The proposition is proved.

Remark. As we shall see in Proposition 5.6, we actually have $T_{\mu}^{\lambda} Q(w \cdot \mu)=Q(w \cdot \lambda)$ when we are in a situation as in the proof above.

Corollary 3.8. Let $\lambda \in X^{+} \backslash C$, and let $E$ be any finite dimensional module in $\mathscr{C}_{k}$. Then each summand of $E \otimes Q(\lambda)$ has quantum dimension zero.

Proof. Combine Proposition 3.5 and Lemma 3.6. 


\section{Applications}

In this section we shall consider $U_{k}$ together with the following family of simple $U_{k}$-modules

$$
\mathscr{F}=\left\{V_{k}(\lambda) \mid \lambda \in C\right\} .
$$

Note that $\mathscr{F}$ consists of irreducible modules for $U_{k}$ which are obtained by deforming irreducible representations of $\mathfrak{L}$. Also the modules in $\mathscr{F}$ have non-zero quantum dimension, see (3.3). In fact, we see that $\mathscr{F}$ is the maximal family with these two properties.

Clearly, $\mathscr{F}$ is stable under duality. In fact, $V_{k}(\lambda)^{*} \cong V_{k}\left(-w_{0} \lambda\right)$ for all $\lambda \in C$. Here $w_{0}$ is the longest element in the Weyl group (so in particular the modules in $\mathscr{F}$ are self-dual for those $\mathfrak{L}$ for which $w_{0}=-1$ ).

Reshetikhin and Turaev [RT] have defined the concept of a modular Hopf algebra as a Hopf algebra with a distinguished family of irreducible representations satisfying certain axioms. We shall not repeat these here but just point out that the following result says that $\left(U_{k}, \mathscr{F}\right)$ satisfies axioms 3 and 4 in the notation from [RT]. Together with the results in loc. cit. and [TW] this then verifies that $\left(U_{k}, \mathscr{F}\right)$ is a modular Hopf algebra and hence produces invariants of 3-manifolds (for quantum $s l_{2}$ axioms 3 and 4 are proved in [RT]).

Corollary 4.1. Let $V_{1}, \ldots, V_{n} \in \mathscr{F}$. Then

$$
V_{1} \otimes \cdots \otimes V_{n}=Z \oplus\left(\bigoplus_{\lambda \in C} V_{k}(\lambda)^{\oplus n_{n}}\right),
$$

where $n_{\lambda} \in \mathbb{N}, \lambda \in C$, and $Z$ is a $U_{k}$-module with the property that $\operatorname{tr}_{q}(\varphi)=0$ for all $\varphi \in \operatorname{End}_{U_{k}}(Z)$.

Proof. From Sect. 2 we know that $V_{1} \otimes \cdots \otimes V_{n}$ is a tilting module and may be written

$$
V_{1} \otimes \cdots \otimes V_{n}=\bigoplus_{\lambda \in X^{+}} Q(\lambda)^{a_{\wedge}}
$$

for certain unique $a_{\lambda} \in \mathbb{N}$. This proves that we may take $n_{\lambda}=a_{\lambda}$ (because $Q(\lambda)=V_{k}(\lambda)$ for $\left.\lambda \in C\right)$ and $Z=\bigoplus_{\lambda \in X^{+} \backslash C} Q(\lambda)^{a_{\Lambda}}$. The required property of $Z$ is then Theorem 3.4.

Our second application has to do with the so-called generalized $6 j$-symbols associated to quantum groups, see [TV] and [DJN]. In order for the above pair $\left(U_{k}, \mathscr{F}\right)$ to fit into the framework of this theory, we need what we shall call a reduced tensor product $\otimes$ on $\mathscr{F}$. Actually, we shall define $\Theta$ on tilting modules:

Let $M$ be a tilting module. Then we define (with notation as in Corollary 2.6)

$$
\bar{M}=\bigoplus_{\lambda \in C} V_{k}(\lambda)^{a_{i}(M)} .
$$

Corollary 2.6 says that the $a_{\lambda}(M)$ are uniquely determined (even by $\operatorname{ch} M$ ) so that $\bar{M}$ is well defined.

Since the tensor product of two tilting modules $M_{1}$ and $M_{2}$ is again a tilting module, we may define

$$
M_{1} \otimes M_{2}=\overline{M_{1} \otimes M_{2}} .
$$


We shall call $\otimes$ the reduced tensor product (because $M_{1} \otimes M_{2}$ is completely reducible).

Corollary 4.2. The reduced tensor product $\otimes$ is associative, i.e. if $M_{1}, M_{2}$ and $M_{3}$ are three tilting modules, then $\left.M_{1} \otimes\left(M_{2} \otimes M_{3}\right) \cong M_{1} \otimes M_{2}\right) \otimes M_{3}$.

Proof. We may write $M_{1} \otimes M_{2}=\left(M_{1} \otimes M_{2}\right) \oplus Z_{1} \quad$ and $\quad M_{2} \otimes M_{3}=$ $\left(M_{2} \otimes M_{3}\right) \oplus Z_{2}$, where $a_{v}\left(Z_{i}\right)=0$ for all $v \in C, i=1,2$. By Corollary 3.8 we have then also $a_{v}\left(E \otimes Z_{i}\right)=0$ for all $v \in C, i=1,2$ and $E$ finite dimensional. It follows that $M_{3} \otimes Z_{1}=0=M_{1} \otimes Z_{2}$. Hence we get

$$
\begin{aligned}
& \left(M_{1} \otimes M_{2}\right) \otimes M_{3} \cong\left(M_{1} \otimes M_{2}\right) \otimes M_{3} \cong \overline{\left(M_{1} \otimes M_{2}\right) \otimes M_{3}} \\
\cong & \overline{M_{1} \otimes\left(M_{2} \otimes M_{3}\right)} \cong M_{1} \otimes\left(M_{2} \otimes M_{3}\right) \cong M_{1} \otimes\left(M_{2} \otimes M_{3}\right) .
\end{aligned}
$$

Remark 4.3. i) The above shows that $\left(U_{k}, \mathscr{F}, \otimes\right)$ satisfies the two first axioms in [DJN, Sect. 2]. By the results in loc. cit. one may thus construct a topological quantum field theory associated to $U_{k}$.

ii) Let us take the opportunity to point out that even though $V_{k}(\lambda) \otimes V_{k}(\mu)$ is a nice semi-simple module for all $\lambda, \mu \in C$, we have not given any formula for determining its irreducible constituents, i.e. we have no general formula for $a_{v}\left(V_{k}(\lambda) \otimes V_{k}(\mu)\right), v, \lambda, \mu \in C$. In fact, the general procedure (contained in Corollary 2.6) for decomposing a tilting module into its indecomposable summands gives explicit answers only if we know $\operatorname{ch} Q(\lambda)$ for all $\lambda \in X^{+}$. By Proposition 5.8 below and [APW2] a solution to this problem would include a solution to the deep problem of determining the irreducible characters $\operatorname{ch} L_{k}(\lambda), \lambda \in X^{+}$(cf. the recent announcement $[\mathrm{KL}])$.

\section{Further Properties of Tilting Modules}

In this section we shall establish some properties of tilting modules not directly related to the main problem studied in the previous sections.

First we shall prove a result (see Proposition 5.6 below) about the behaviour of tilting modules under translations.

Fix $\lambda \in C$ and $\mu \in \bar{C}$ with $\operatorname{stab}_{W_{l}}(\mu)=\{1, s\}$. In this case, recall that we have (see [APW1, Sect. 8])

$$
T_{\lambda}^{\mu} H_{k}^{0}(y \cdot \lambda)=H_{k}^{0}(y \cdot \mu) \text { for all } y \in W_{l},
$$

For any $y \in W_{l}$ with $y s \cdot \lambda<y \lambda$ and $y \cdot \mu \in X^{+}$, we have a non-split exact sequence

$$
0 \rightarrow H_{k}^{0}(y s \cdot \lambda) \rightarrow T_{\mu}^{\lambda}\left(H_{k}^{0}(y \cdot \mu)\right) \rightarrow H_{k}^{0}(y \cdot \lambda) \rightarrow 0 .
$$

Lemma 5.3. For $\lambda$ and $\mu$ as above we have

$$
T_{\lambda}^{\mu} T_{\mu}^{\lambda} Q(y \cdot \mu) \cong Q(y \cdot \mu) \oplus Q(y \cdot \mu)
$$

for all $y \in W_{l}$ with $y \cdot \mu \in X^{+}$. 
Proof. Since $T_{\lambda}^{\mu} T_{\mu}^{\lambda} Q(y \cdot \mu)$ is a tilting module, we see from Corollary 2.6 that it is enough to prove that the two sides have the same characters. But this follows because by (5.1) and (5.2) we get $T_{\lambda}^{\mu} T_{\mu}^{\lambda} H_{k}^{0}(w \cdot \mu) \cong H_{k}^{0}(w \cdot \mu) \oplus H_{k}^{0}(w \cdot \mu)$ for all $w \in W_{l}$.

Lemma 5.4. Let $\lambda \in C, v \in X^{+}$and $y \in W_{l}$ with $y \cdot \lambda \in X^{+}$. If $L_{k}(v)$ is a composition factor of $Q(y \cdot \lambda)$, then $v$ is strongly linked to $y \cdot \lambda$.

Proof. We proceed by induction on $y$. If $y=1$, we have $Q(\lambda)=L_{k}(\lambda)$ and the lemma is trivial. Suppose $y \neq 1$ and pick $s$ such that $y s \cdot \lambda<y \cdot \lambda$. Let us first prove that the lemma is true if we replace $Q(y \cdot \lambda)$ by $T_{\mu}^{\lambda} T_{\lambda}^{\mu} Q(y s \cdot \lambda)$. By the strong linkage principle (1.2), it is enough to prove the statement for those $v$ for which $H_{k}^{0}(v)$ occurs in a good filtration for $T_{\mu}^{\lambda} T_{\lambda}^{\mu} Q(y s \cdot \lambda)$. But by (5.1) and (5.2) such a $v$ has the form $w \cdot \lambda$ or $w s \cdot \lambda$ for some $w \in W_{l}$ for which $H_{k}^{0}(w \cdot \lambda)$ occurs in a good filtration of $Q\left(y_{s} \cdot \lambda\right)$. By induction hypothesis, $w \cdot \lambda$ is strongly linked to $y s \cdot \lambda$ and hence to $y \cdot \lambda$. Moreover, $w \cdot \lambda$ strongly linked to $y s \cdot \lambda<y \cdot \lambda$ implies that $w s \cdot \lambda$ is strongly linked to $y \cdot \lambda$.

Finally, to obtain the lemma for $Q(y \cdot \lambda)$ we just observe that $T_{\mu}^{\lambda} T_{\lambda}^{\mu} Q(y s \cdot \lambda)$ is a tilting module. The above shows that $y \cdot \lambda$ is its unique highest weight. Hence $Q(y \cdot \lambda)$ is a direct summand, and the lemma follows.

Lemma 5.5. Let $\lambda, \mu$ and $s$ be as above. Suppose $y \in W_{l}$ with $y \cdot \mu \in X^{+}$and $y s \cdot \lambda<y \cdot \lambda$. Then $H_{k}^{0}(y s \cdot \lambda)$ occurs at least once in a good filtration of $Q(y \cdot \lambda)$.

Proof. Consider the diagram

$$
\begin{array}{ccc}
0 \rightarrow \quad Q^{\prime} & \rightarrow \quad Q(y \cdot \lambda) & \rightarrow H_{k}^{0}(y \cdot \lambda) \rightarrow 0 \\
& & \| \\
0 \rightarrow H_{k}^{0}(y s \cdot \lambda) & \rightarrow T_{\mu}^{\lambda} H_{k}^{0}(y \cdot \mu) & \rightarrow H_{k}^{0}(y \cdot \lambda) \rightarrow 0,
\end{array}
$$

where the first sequence is the top piece of a good filtration for $Q(y \cdot \lambda)$ and the second sequence is (5.2). The dotted map exists (making the diagram commutative) because by (1.4) we have $\operatorname{Ext}_{\mathscr{C}_{k}}^{1}\left(Q(y \cdot \lambda), H_{k}^{0}(y s \cdot \lambda)\right)=0$. The induced map $Q^{\prime} \rightarrow H_{k}^{0}(y s \cdot \lambda)$ is non-zero because otherwise we would have a splitting of (5.2). This means that $H_{k}^{0}(y s \cdot \lambda)$ must occur (at the top) in a good filtration of $Q^{\prime}$ because by Lemma $5.4 y s \cdot \lambda$ is maximal for the strong linkage ordering among the high weights of composition factors of $Q^{\prime}$.

Proposition 5.6. For $\lambda$ and $\mu$ as above we have

$$
T_{\mu}^{\lambda} Q(y \cdot \mu) \cong Q(y \cdot \lambda)
$$

for all $y \in W_{l}$ with $y \cdot \mu \in X^{+}$and $y s \cdot \lambda<y \cdot \lambda$.

Proof. It is clear from Lemma 5.4 that $T_{\lambda}^{\mu} Q(y s \cdot \lambda)$ contains $Q(y \cdot \mu)$ as a summand and hence that the composition factors of $Q(y \cdot \mu)$ have highest weights strongly linked to $y \cdot \lambda$. It follows that $T_{\mu}^{\lambda} Q(y \cdot \mu)$ has highest weight $y \cdot \lambda$. Hence $T_{\mu}^{\lambda} Q(y \cdot \mu)=Q(y \cdot \lambda) \oplus Q$ for some tilting module $Q$. From Lemma 5.5 we see that $T_{\lambda}^{\mu} Q(y \cdot \lambda)$ has $H_{k}^{0}(y \cdot \mu)$ occurring (at least) twice in a good filtration. Moreover, by Lemma 5.4 we have that $y \cdot \mu$ is a highest weight of $T_{\lambda}^{\mu} Q(y \cdot \lambda)$. We conclude that $Q(y \cdot \mu) \oplus Q(y \cdot \mu)$ is a summand of $T_{\lambda}^{\mu} Q(y \cdot \lambda)$. Comparing this with Lemma 5.3 we see that $T_{\lambda}^{\mu} Q=0$. But the $H_{k}^{0}(x \cdot \lambda)$ 's occurring in a good filtration of $Q$ are among those occurring in a good filtration for $T_{\mu}^{\lambda} Q(y \cdot \mu)$ and all of these satisfy $T_{\lambda}^{\mu} H_{k}^{0}(x \cdot \lambda) \neq 0$. Hence $Q=0$. 
Secondly, we shall prove that "most" indecomposable tilting modules are injective/projective in $\mathscr{C}_{k}$.

Recall from [APW1, Sect. 9] that the injective modules in $\mathscr{C}_{k}$ may be obtained as summands of $\mathrm{St}_{k} \otimes E$ with $E$ running through a set of finite dimensional $U_{k}$-modules. Here $\mathrm{St}_{k}=L_{k}((l-1) \rho)=H_{k}^{0}((l-1) \rho)=V_{k}((l-1) \rho) \quad$ is the Steinberg module. By examining the arguments in loc. cit. it is easy to see that the $E$ 's may be chosen among the tilting modules in $\mathscr{C}_{k}$. Hence

All injective modules in $\mathscr{C}_{k}$ are tilting modules .

Now, let for $\lambda \in X^{+}$the injective hull in $\mathscr{C}_{k}$ of $L_{k}(\lambda)$ be denoted $I_{k}(\lambda)$. If $\lambda=\lambda_{0}+l \lambda_{1}$, with $0 \leqq\left\langle\lambda_{0}, \alpha^{v}\right\rangle<l$ for all simple roots, then we set $\bar{\lambda}=2(l-1) \rho+w_{0} \lambda_{0}+\bar{l} \lambda_{1}$ (compare [D2]).

Proposition 5.8. For all $\lambda \in X^{+}$we have $I_{k}(\lambda)=Q(\bar{\lambda})$.

Proof. By the above $I_{k}(\lambda)$ is an indecomposable tilting module. By construction (see [APW1, Sect. 9]) it has highest weight $\lambda$. The proposition follows.

Remark. This proposition shows that the quantum analogue of Donkin's conjecture in [D2, Sect. 2] holds.

Corollary 5.9. Let $v \in X^{+}$and suppose $\left\langle v, \alpha^{\vee}\right\rangle \geqq l$ for all simple roots $\alpha$. Then $Q(v)$ has simple head and simple socle.

Corollary 5.10. In the above notation we have

$$
Q(\bar{\lambda}) \cong Q\left(\bar{\lambda}_{0}\right) \otimes L_{k}\left(l \lambda_{1}\right)
$$

for all $\lambda \in X^{+}$.

Proof. By [APW2] we know that $I_{k}(\lambda) \cong I_{k}\left(\lambda^{0}\right) \otimes L_{k}\left(l \lambda_{1}\right)$. Hence the corollary is immediate from Proposition 5.8.

Corollary 5.11. Let $v \in X^{+}$with $\left\langle v, \alpha^{v}\right\rangle \geqq l$ for all simple roots $\alpha$. Then for each finite dimensional module $E \in \mathscr{C}_{k}$ we have

$$
Q(v) \otimes E \text { is a tilting module . }
$$

Furthermore, if $a_{\lambda}(Q(v) \otimes E) \neq 0$, then $\left\langle\lambda, \alpha^{\vee}\right\rangle \geqq l-1$ for all simple roots $\alpha$.

Proof. Since injectivity is preserved when tensoring by $E$, the corollary follows from Proposition 5.8.

Applying the same argument to the tilting module $\mathrm{St}_{k}$, we obtain a quantum analogue of another conjecture by Donkin stated in a talk at MSRI in November 1990:

Corollary 5.12. For any finite dimensional module $E \in \mathscr{C}_{k}$ we have

$$
\mathrm{St}_{k} \otimes E \text { is a tilting module }
$$

and if $a_{\lambda}(\mathrm{St} \otimes E) \neq 0$, then $\left\langle\lambda, \alpha^{\vee}\right\rangle \geqq l-1$ for all simple roots $\alpha$. 


\section{References}

[APW1] Andersen, H.H., Polo, P., Wen, K.: Representations of quantum algebras. Invent. Math. 104, 1-59 (1991)

[APW2] Andersen, H.H., Polo, P., Wen, K.: Injective modules for quantum algebras. Am. J. Math. 114, 571-604 (1992)

[AW] Andersen, H.H., Wen, K.: Representations of quantum algebras. The mixed case. J. Reine Angew. Math. 427, 35-50 (1992)

[CPSvdK] Cline, E., Parshall, B., Scott, L., van der Kallen, W.: Rational and generic cohomology. Invent. Math. 39, 129-165 (1980)

[D1] Donkin, S.: Rational representations of algebraic groups. Lecture Notes in Math. vol. 1140, Berlin Heidelberg, New York: Springer 1985

[D2] Donkin, S.: On tilting modules for algebraic groups. Preprint QMW (1991)

[DJN] Durhuus, B., Jakobsen, P., Nest, R.: Topological Quantum Field Theories from Generalized 6J-Symbols. Preprint No. 11 (1991), Københavns University

[J] Jantzen, J.C.: Representations of algebraic groups. Pure Appl. Math., vol. 131, London, New York: Academic Press 1987

[KL] Kazhdan, D., Lusztig, G.: Affine Lie algebras and quantum groups. Internat. Math. Research Notes (Duke Math. J.) 2, 21-29 (1991)

[KM] Kirby, R., Melvin, P.: The 3-manifold invariants of Witten and Reshetikhin-Turaev for $s l(2, C)$. Invent. Math. 105, 473-545 (1991)

[L1] Lusztig, G.: Quantum groups at roots of 1. Geom. Dedicata 35, 89-114 (1990)

[L2] Lusztig, G.: Canonical bases arising from quantized enveloping algebras II. Progr. Phys. Suppl. 102, 175-202 (1990)

[M] Mathieu, O.: Filtrations of $G$-modules. Ann. Sci. École Norm. Sup. 23, 625-644 (1990)

[P] Paradowski, J.: Filtrations of modules over quantum algebras. Preprint 1992

[R] Ringel, C.M.: The category of modules with good filtrations over a quasi-hereditary algebra has almost split sequences. Math. Z. 208, 209-223 (1991)

[RT] Reshetikhin, N., Turaev, V.: Invariants of 3-manifolds via link polynomials and quantum groups. Invent. Math. 103, 547-597 (1991)

[T] Thams, L.: Two classical results in the quantum mixed case. J. Reine Angew. Math. (to appear)

[TV] Turaev, V., Viro, O.: State sum invariants of 3-manifolds and quantum 6j-symbols. Preprint 1991

[TW] Turaev, V., Wenzl, H.: Quantum invariants of 3-manifolds associated with classical simple Lie algebras. Preprint 1991

[W] Wang, J-p.: Sheaf cohomology of $G / B$ and tensor products of Weyl modules. J. Algebra 77, 162-185 (1982)

Communicated by K. Gawedzki 
